\title{
Fluorescence Measurements of Expanding Strongly Coupled Neutral Plasmas
}

\author{
E. A. Cummings \\ J. E. Daily \\ Dallin S. Durfee \\ Scott D. Bergeson \\ scott.bergeson@byu.edu
}

Follow this and additional works at: https://scholarsarchive.byu.edu/facpub

Part of the Astrophysics and Astronomy Commons, and the Physics Commons

\section{Original Publication Citation}

E. A. Cummings, J. E. Daily, D. S. Durfee, and S. D. Bergeson, “Fluorescence measurement of expanding strongly-coupled neutral plasmas," Physical Review Letters, 95, 2351 (25). The original publication of this article can be found at http://link.aps.org/doi/1.113/ PhysRevLett.95.2351

\section{BYU ScholarsArchive Citation}

Cummings, E. A.; Daily, J. E.; Durfee, Dallin S.; and Bergeson, Scott D., "Fluorescence Measurements of Expanding Strongly Coupled Neutral Plasmas" (2005). Faculty Publications. 337.

https://scholarsarchive.byu.edu/facpub/337

This Peer-Reviewed Article is brought to you for free and open access by BYU ScholarsArchive. It has been accepted for inclusion in Faculty Publications by an authorized administrator of BYU ScholarsArchive. For more information, please contact ellen_amatangelo@byu.edu. 


\title{
Fluorescence Measurements Of Expanding Strongly Coupled Neutral Plasmas
}

\author{
E. A. Cummings, ${ }^{*}$ J.E. Daily, D. S. Durfee, and S. D. Bergeson ${ }^{\dagger}$ \\ Brigham Young University, Department of Physics and Astronomy, Provo, Utah 84602, USA
}

(Received 8 June 2005; published 29 November 2005)

\begin{abstract}
We report new detailed density profile measurements in expanding strongly coupled neutral calcium plasmas. Using laser-induced fluorescence techniques, we determine plasma densities in the range of $10^{5}$ to $10^{9} \mathrm{~cm}^{-3}$ with a time resolution limit as small as $7 \mathrm{~ns}$. Strong coupling in the plasma ions is inferred directly from the fluorescence signals. Evidence for strong coupling at late times is presented, confirming a recent theoretical result.
\end{abstract}

DOI: 10.1103/PhysRevLett.95.235001

Strongly coupled Coulomb systems appear in extreme conditions, such as in quark-gluon plasmas [1], in laserfusion plasmas [2], and in some astrophysical settings. A new class of strongly interacting neutral plasmas was recently demonstrated using the tools of laser cooling and trapping [3-7]. These "ultracold" neutral plasmas occupy a unique position in phase space. In these plasmas it is possible to create strongly interacting Coulomb systems at modest densities because the initial electron and ion temperatures can be in the milli-Kelvin range. The initial ion-ion and ion-electron interaction strength can also be selected with great precision.

Recent experimental work in this field has used absorption imaging techniques to make temperature and density measurements in expanding ultracold neutral plasmas $[6,7]$. This work explored the 50 to $1000 \mathrm{~ns}$ time period after plasma formation in great detail. Correlation-induced heating was observed in the plasma ions. The ion coupling parameter, given as the ratio of nearest-neighbor Coulomb energy to the kinetic energy, equilibrated just inside the strongly coupled regime, with a coupling parameter around 2. Radio-frequency (rf) excitation techniques have also been used to determine the average ion density and the electron temperature in these systems $[4,8,9]$. These studies confirm theoretical predictions regarding the generally self-similar Gaussian expansion of the ions and the clamping of the electron temperature in the weakly coupled regime.

In this Letter we report laser-induced-fluorescence measurements of ions in expanding strongly coupled plasmas as a tool to study the spatial and temporal evolution of the ion temperature and density. This measurement technique has a $7 \mathrm{~ns}$ temporal resolution limit. We measure plasma densities as low as $10^{5} \mathrm{~cm}^{-3}$ at effective plasma temperatures of $100 \mathrm{~K}$. The maximum density that can be measured is limited by radiation trapping, and for spherically symmetric systems in the milli-Kelvin range is limited to around $10^{9} \mathrm{~cm}^{-3}$. The temporal resolution and dynamic range of this method in ultracold plasma measurements surpass those currently seen in absorption spectroscopy, and rival the sensitivity of $\mathrm{rf}$ spectroscopic methods. Contrary to earlier work, we find no evidence for a shock
PACS numbers: 52.27.Gr, 32.80.Pj, 52.27.Cm, $52.70 . \mathrm{Kz}$

front in the expansion, and we observe density modulations that are not "frozen" into the distribution [10]. We also show that the ion strong-coupling parameter is well above 0.2 , which was once believed to be the limit in the long time expansion [3-6,10-13], and that is also higher than reported in recent absorption measurements [7].

We create a calcium magneto-optical trap (MOT) using the resonance transition at $423 \mathrm{~nm}$. Up to $50 \mathrm{~mW}$ of $423 \mathrm{~nm}$ radiation is generated by frequency doubling a diode laser system at $846 \mathrm{~nm}$ using a periodically poled potassium titanyl phosphate crystal in a resonant buildup cavity [14]. The $423 \mathrm{~nm}$ MOT light is detuned one linewidth below the atomic transition, making the MOT temperature around $1 \mathrm{mK}$. The density distribution is approximately Gaussian in $x, y$, and $z$, of the form

$$
n=n_{0} \exp \left[-\left(x^{2}+y^{2}\right) / \sigma_{0}^{2}-z^{2} / \beta_{0}^{2}\right] .
$$

The $1 / e^{2}$ radius is $\sigma_{0}=0.5 \mathrm{~mm}$ in the $x$ and $y$ dimensions, and $\beta_{0}=2.5 \sigma_{0}=1.25 \mathrm{~mm}$ in the $z$ dimension. We use a grating-stabilized $672 \mathrm{~nm}$ diode laser as a repumper to increase the number of atoms in the trap. The peak density is $4 \times 10^{9} \mathrm{~cm}^{-3}$.

We photoionize the atoms in the MOT using a two-color, two-photon ionization process. A portion of the $846 \mathrm{~nm}$ diode laser radiation from the MOT laser is pulse amplified in a pair of yttrium-alluminum-garnet (YAG) laser pumped dye cells and frequency doubled. This produces a 3 nsduration laser pulse at $423 \mathrm{~nm}$ with a pulse energy around $1 \mu \mathrm{J}$. This laser pulse passes through the MOT, and its peak intensity is a few thousand times greater than the saturation intensity. A second YAG-pumped dye laser at $390 \mathrm{~nm}$ counter propagates the $423 \mathrm{~nm}$ pulse and excites the MOT atoms to low-energy states above the ionization potential. We photoionize $85 \%-90 \%$ of the ground-state atoms in the MOT. The minimum electron temperature is limited by the $30 \mathrm{GHz}$ bandwidth of the $390 \mathrm{~nm}$ laser to about $1 \mathrm{~K}$.

Ions in the plasma scatter light from a probe laser beam tuned to the Ca II ${ }^{2} S_{1 / 2}-{ }^{2} P_{1 / 2}$ transition at $397 \mathrm{~nm}$. The probe laser is generated by a grating-stabilized violet diode laser locked to the calcium ion transition using the dichroic atomic vapor laser lock technique [15] in a large-bore, low- 
pressure hollow cathode discharge of our own design. The probe laser is spatially filtered and a few $\mu \mathrm{W}$ of power is focused to a Gaussian waist of $130 \mu \mathrm{m}$ in the MOT. We average repeated measurements of the scattered $397 \mathrm{~nm}$ radiation with the probe laser in a given position. This produces a time-resolved signal proportional to the number of atoms resonant with the probe beam in a particular column of the plasma. By translating a mirror just outside the MOT chamber, we scan the probe laser across the ion cloud. In this manner we obtain temporal and spatial information about the plasma expansion. Typical fluorescence measurements are shown in Fig. 1.

When the probe beam is on axis (Fig. 1, top panel), the fluorescence signal takes roughly $20 \mathrm{~ns}$ to reach its maximum value. This duration is a few times the $7 \mathrm{~ns}$ radiative lifetime of the $\mathrm{Ca} \mathrm{II}{ }^{2} P_{1 / 2}$ level. This is an example of the classic Rabi two-level atom problem with spontaneous emission, where a collection of atoms driven on resonance will reach a steady excited-state population after a few ra-

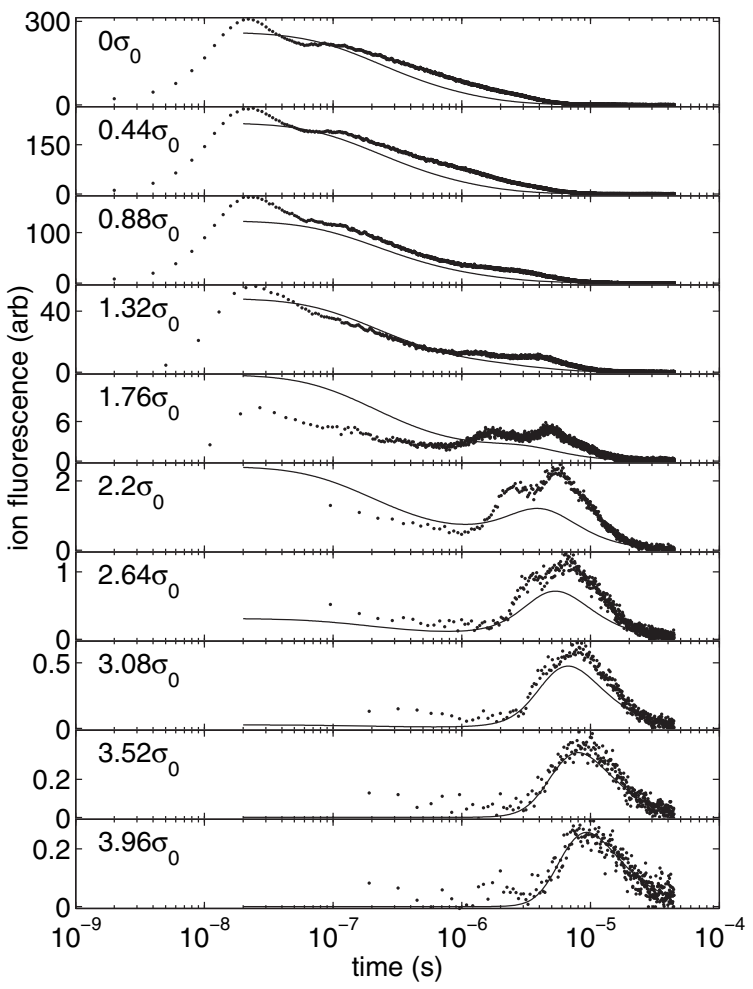

FIG. 1. Laser-induced fluorescence of ions in an expanding ultracold plasma. In the upper panel, the probe beam is parallel to (and collinear with) the long axis of the initial plasma density distribution. In each successive panel, the probe beam is moved $0.22 \mathrm{~mm}=0.44 \sigma_{0}$ farther away from this symmetry axis, where $\sigma_{0}$ is the $1 / e^{2}$ transverse Gaussian radius of the initial plasma density distribution in the $x$ dimension. Note that the time scale is logarithmic and the fluorescence scale is linear. The solid line is the expected signal from a thermal expansion model, as explained in the text. The detuning of the ionization laser above threshold is $58 \mathrm{~cm}^{-1}$. diative lifetimes. We also see this same turn-on time when we perform absorption measurements on the plasma ions.

After the signal peaks, it rapidly decays. At $t \sim 100 \mathrm{~ns}$ the decay slows down. This is due to correlation-induced heating in the ions, similar to previously published absorption measurements [7]. During the first microsecond of the decay, while the spatial distribution of the plasma ions has not changed, it is straightforward to convert this fluorescence decay signal into velocity as a function of time. With a few assumptions, we can determine the temperature, density, and strong-coupling parameter of the plasma ions.

An atom moving with velocity $v_{z}$ parallel to the propagation direction of the probe laser beam has a Lorentzian probability of scattering a photon, proportional to $\left[\left(v_{z} / b\right)^{2}+1\right]^{-1}$. The constant $b=\gamma \lambda / 2$ is the velocity that corresponds to a Doppler shift equal to the natural linewidth, $\gamma=1 / 2 \pi \tau$ is the natural linewidth of the transition, and $\lambda$ is the transition wavelength. The initial velocity distribution is Maxwellian, of the form $\exp \left(-v_{z}^{2} / 2 v_{\mathrm{th}}^{2}\right) / \sqrt{\pi} v_{\text {th }}$. For systems in thermal equilibrium, $v_{\text {th }}$ is the thermal velocity. As mentioned in the literature [7], the temperature should be a measure of the random motion of ions. In these kinds of measurements for expanding ultracold plasmas, this random motion is observable only at early times. The random motion is quickly overwhelmed by the directed motion of the plasma expansion. So $v_{\text {th }}$ quickly looses its meaning in a thermodynamic sense. However, when the ions are "heated" by correlations the Maxwellian approximation is still valid.

We integrate the product of the Lorentzian line shape and the Maxwellian distribution to get an expression for how the fluorescence signal, $s(t)$, changes with the width of the ion velocity distribution as a function of time:

$$
s(t) \propto \frac{1}{v_{\text {th }}} \exp \left(\frac{b^{2}}{2 v_{\text {th }}^{2}}\right) \operatorname{erfc}\left(\frac{b}{\sqrt{2} v_{\text {th }}}\right),
$$

where erfc $(x)$ is the complimentary error function of the parameter $x$. If $v_{\text {th }}$ is known at a particular time, this equation can be inverted to give the change in the thermal velocity as a function of time. The initial neutral atom velocity is near the Doppler limit at $40 \mathrm{~cm} / \mathrm{s}$. The neutral atoms also experience an ionization recoil velocity of $40 \mathrm{~cm} / \mathrm{s}$. In the inversion of Eq. (2), we set the scale so that a linear extrapolation of the signal to time $t=0$ gives the quadrature sum of the Doppler and recoil velocities, $56 \mathrm{~cm} / \mathrm{s}$, as shown in Fig. 2.

A thermal model of ultracold plasma expansions was presented in Refs. [10,11]. The directed expansion velocity is

$$
v(r, t)=\frac{v_{e}^{2} t r}{\sigma^{2}(t)}
$$

where $\sigma(t)=\sqrt{\sigma_{0}^{2}+v_{e}^{2} t^{2}}$ is the time-dependent width of a self-similar Gaussian expansion, and $v_{e}$ is the asymptotic electron velocity [8]. At early times, when $v_{e}^{2} t^{2} \ll \sigma_{0}^{2}$, the velocity reduces to $v(r, t) \approx v_{e}^{2} t r / \sigma_{0}^{2}$. 


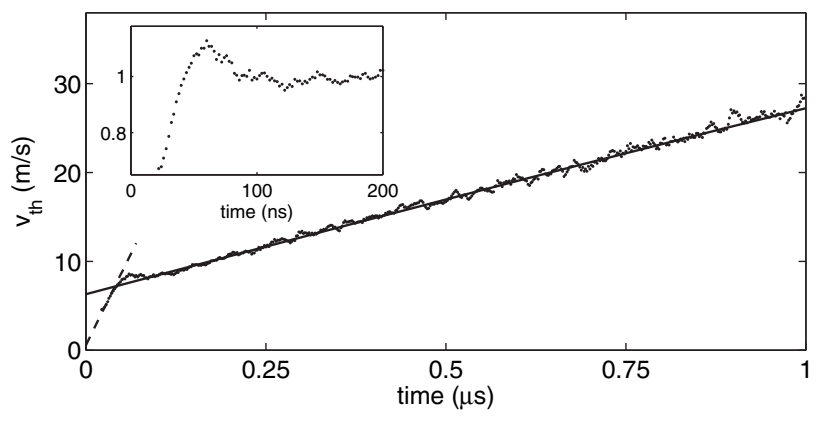

FIG. 2. The mean $z$ component of the ion velocity as a function of time after plasma formation. The points show the velocity extracted from the ion fluorescence signal shown in the top panel of Fig. 1. The solid line is a fit of the data to a thermal model [11], as described in the text. The dashed line shows the extrapolation to $56 \mathrm{~cm} / \mathrm{s}$ at time $t=0$. The inset shows the ratio of the data to the thermal model, accentuating the correlation-induced heating signal.

In applying this expression for the expansion velocity to the data in Fig. 2, some care is required. The model assumes ions initially at rest. However, in our experiment the plasma ions have an initial electrical potential energy which is quickly converted to kinetic energy (see the discussion of correlation heating below). This gives an offset to the $t=0$ velocity predicted by the model.

Otherwise, the application is straightforward. The probe laser interacts with ions in a particular volume. During the first microsecond, the large-scale ion distribution does not change, even though the ions are accelerating. The expression in Eq. (3) predicts a velocity that depends linearly in time, with $v_{e}^{2} r / \sigma_{0}^{2}$ averaged over the part of the density distribution interacting with the probe laser beam. A fit of our data to this model is shown in Fig. 2. For the early expansion times, the lack of spherical symmetry in our plasma is unimportant in fitting this data, because the average $v_{e}^{2} r / \sigma_{0}^{2}$ appears as a fit parameter.

This figure illustrates correlation-induced heating, and provides an independent check of the plasma density. When the plasma is created, the ions quickly move from their initial disordered state into a state with greater order. This motion is initially synchronized at the plasma frequency. But because of variations in the local electric field at each ion, the oscillatory motion becomes unsynchronized. The time from plasma creation to the maximum temperature (minimum signal) in this oscillation is equal to $t=1 / 4 \omega_{p}$, where $\omega_{p}$ is the average plasma frequency. As seen in the inset to Fig. 2, the peak occurs at $60 \mathrm{~ns}$, giving an average plasma density of $4.0 \times 10^{8} \mathrm{~cm}^{-3}$, about a factor of 3 lower than what we expect based on the ionization fraction of the neutral MOT. The cause of this discrepancy is not readily apparent. Our numerical modeling of the plasma oscillation dynamics indicates that the average density determined from the observed oscillation period reproduces the average plasma density to within $20 \%$. Perhaps this discrepancy indicates the number of plasma ions that have recombined into highlying Rydberg states at early times.

The strong-coupling constant in this system can be determined from data extracted from Fig. 2. The kinetic energy of the system is equal to $m v^{2} / 2$, where $v^{2}=v_{x}^{2}+$ $v_{y}^{2}+v_{z}^{2}$, and the random thermal motion due to correlation heating is assumed to be isotropic. The temperature is determined from $3 k_{b} T / 2=m v^{2} / 2$. Using the derived values of $v_{z}=8 \mathrm{~m} / \mathrm{s}$ and $n=4 \times 10^{8} \mathrm{~cm}^{-3}$, the strongcoupling parameter is $\Gamma=\left(e^{2} / 4 \pi \epsilon_{0} k_{B} T\right)(4 \pi n / 3)^{1 / 3}=$ 4. This value of the coupling parameter is a factor of 2 higher than previously measured in $\mathrm{Sr}$ plasmas [7].

Early simulations [10-13] predicted that the electron coupling parameter would be clamped at $\Gamma \sim 0.2$, limited mainly by a balance between three-body recombination and adiabatic cooling. Because of energy equipartition in this neutral plasma, the ions would also have this same $\Gamma$ at equilibrium. However, recent work [16] shows that before the ions equilibrate with the electrons, the relaxation time scale exceeds the hydrodynamic time scale, and full equilibrium is never reached. This allows the ions to remain strongly coupled with $\Gamma>1$ throughout the expansion.

During the time from 1 to $40 \mu \mathrm{s}$, the fluorescence signal depends on the time evolution of the ion temperature, the overall plasma expansion velocity, and the density. As the plasma expands, the number of ions in the probe laser beam decreases. Also, as the velocity distribution widens, the fraction of atoms in the beam that are in resonance with the probe laser frequency also decreases.

The thermal expansion model in Ref. [11] provides a theoretical framework for interpreting our data. Most of the ions in the plasma lie in a region in which the electric field depends linearly on the spatial coordinate. The ansatz presented in Ref. [10] simplifies the system by imposing this linear dependence of the electric field for the entire system. While this is clearly not true for ions near the edge of the plasma, the number of ions in that region is small. Sophisticated approaches to modeling this system have shown that the thermal model captures the major features of the expansion dynamics $[10,11,17]$.

The lack of spherical symmetry in our experiments changes the slope of the electric field in the $z$ direction relative to $x$ (or $y$ ), because the plasma distribution is elongated in $z$ by a factor of 2.5 [see Eq. (1)]. Keeping with the ansatz of Ref. [10], we assume that the Gaussian plasma expands in a self-similar fashion. Similar to Eq. (3), the expansion velocity in the $x$ direction is $v_{x}(t)=v_{e}^{2} t x / \sigma^{2}(t)$. The expansion velocity in the $z$ direction is $v_{z}(t)=v_{e}^{2} t z / \beta^{2}(t)$, where $\beta=\sqrt{\left(2.5 \sigma_{0}\right)^{2}+v_{e}^{2} t^{2}}$. This result, which relates the velocity to the position, will be important in modeling the fluorescence signals, as discussed below.

In the experiment, the probe laser beam is spatially filtered and focused into the plasma with a Gaussian beam waist of $w=130 \mu \mathrm{m}$. The position offset of this probe laser relative to the plasma is denoted by the distance 
$a$. The two-dimensional convolution of the probe laser and the plasma distribution is proportional to the number of plasma ions per unit length along the probe beam,

$$
\eta=\frac{w^{2}}{\beta\left(w^{2}+2 \sigma^{2}\right)} \exp \left(-\frac{2 a^{2}}{w^{2}+2 \sigma^{2}}\right) \exp \left(-\frac{z^{2}}{\beta^{2}}\right) .
$$

Multiplying this expression by the Lorentzian line shape gives a probability that an ion in the probe beam column moving with a $z$ component of velocity $v_{z}$ interacts with the probe laser. We substitute for $v_{z}$ the expression derived previously, and integrate Eq. (4) over $z$ to derive the predicted time dependence of the fluorescence signal:

$$
s(t) \propto \operatorname{erfc}\left(\frac{l}{\beta}\right) \frac{w^{2} l}{\beta\left(w^{2}+2 \sigma^{2}\right)} \exp \left(-\frac{2 a^{2}}{w^{2}+2 \sigma^{2}}+\frac{l^{2}}{\beta^{2}}\right),
$$

where $l \equiv \gamma \lambda \sigma^{2}(t) / 2 t v_{e}^{2}$. This derivation neglects the influence of the thermal velocity, and is valid at times where the directed expansion velocity dominates the thermal velocity [a regime opposite to that for Eq. (2)]. Adding the thermal velocity to the model would further reduce the predicted signal levels at early times.

This fluorescence model is the solid line plotted in Fig. 1, with $v_{e}$ and the overall amplitude as fit parameters. They are chosen so that the model agrees with the data in the last panel of the figure. Considering the simplicity of this model, it is surprising that it matches the general shape and order of magnitude of the fluorescence signal. Relative to the model, the experimental data are consistently higher at later times. Additionally, data recorded with the probe laser beam between $a=\sigma_{0}$ and $3 \sigma_{0}$ show structures that cannot be explained by the model. Predictions of ionacoustic waves freezing into the plasma expansion have been made. However, the observed modulations are not stationary relative to the plasma expansion. A vertical slice through all of the panels in Fig. 1 gives the density profile at a given time. The data show that two local maxima that develop in the interior of the plasma coalesce into one another and disappear relative to the smooth Gaussian shape predicted by the model (lowest frame). Contrary to predictions, we see no evidence for shock formation in these plasmas.

The plasma ions are strongly coupled throughout the expansion. The ions equilibrate after $100 \mathrm{~ns}$ with a coupling parameter of $\Gamma=4$. Recent hybrid molecular dynamics calculations [16] show that after an initial oscillation, the coupling parameter always increases. The absence of a shock front in the plasma expansion and also the observed suppression of local density variations gives evidence that the plasma ion system is collisionally stiff, indicative of strong coupling.

In conclusion, we have demonstrated a new technique for determining the density of ions in an expanding ultracold plasma using fluorescence spectroscopy. Our implementation of this technique has a time resolution of $7 \mathrm{~ns}$. We report measurements of the density profile for up to $50 \mu \mathrm{s}$, and demonstrate a sensitivity limit around
$10^{5} \mathrm{~cm}^{-3}$. This method surpasses the time resolution and dynamic range of previously reported techniques. It rivals the sensitivity limit of $\mathrm{rf}$ techniques, and provides detailed spatial information of the plasma density profile. We have created a plasma inside the strongly coupled regime, and observed changes in the plasma density over time. These plasmas are strongly coupled for the entire measured expansion. The general features of our measurements are in reasonable agreement with a thermal model, although the remaining discrepancies require more sophisticated methods to interpret quantitatively. We find no evidence for a shock front in the expansion, and density modulations that are not stationary with respect to the scaled density distribution.

This research is supported in part by Brigham Young University, the Research Corporation, and the National Science Foundation (Grant No. PHY-9985027).

*Present address: Lockheed Martin Space Systems Company, Sunnyvale, CA 94089.

†Electronic address: scott.bergeson@byu.edu

[1] Edward V. Shuryak and Ismail Zahed, Phys. Rev. C 70, 021901 (2004).

[2] Nigel C. Woolsey, David Riley, and Eran Nardi, Rev. Sci. Instrum. 69, 418 (1998).

[3] T. C. Killian, S. Kulin, S. D. Bergeson, L. A. Orozco, C. Orzel, and S.L. Rolston, Phys. Rev. Lett. 83, 4776 (1999).

[4] S. Kulin, T. C. Killian, S. D. Bergeson, and S. L. Rolston, Phys. Rev. Lett. 85, 318 (2000).

[5] T. C. Killian, M. J. Lim, S. Kulin, R. Dumke, S. D. Bergeson, and S. L. Rolston, Phys. Rev. Lett. 86, 3759 (2001).

[6] C.E. Simien, Y.C. Chen, P. Gupta, S. Laha, Y.N. Martinez, P. G. Mickelson, S. B. Nagel, and T. C. Killian, Phys. Rev. Lett. 92, 143001 (2004).

[7] Y.C. Chen, C.E. Simien, S. Laha, P. Gupta, Y.N. Martinez, P. G. Mickelson, S. B. Nagel, and T. C. Killian, Phys. Rev. Lett. 93, 265003 (2004).

[8] S. D. Bergeson and R. L. Spencer, Phys. Rev. E 67, 026414 (2003).

[9] J.L. Roberts, C. D. Fertig, M. J. Lim, and S. L. Rolston, Phys. Rev. Lett. 92, 253003 (2004).

[10] F. Robicheaux and James D. Hanson Phys. Rev. Lett. 88, 055002 (2002).

[11] F. Robicheaux and James D. Hanson, Phys. Plasmas 10, 2217 (2003).

[12] S. G. Kuzmin and T. M. O’Neil, Phys. Rev. Lett. 88, 065003 (2002).

[13] S. G. Kuzmin and T. M. O’Neil, Phys. Plasmas 9, 3743 (2002).

[14] A. D. Ludlow, H. M. Nelson, and S. D. Bergeson, J. Opt. Soc. Am. B 18, 1813 (2001).

[15] Kristan L. Corwin, Zheng-Tian Lu, Carter F. Hand, Ryan J. Epstein, Carl E. Wieman, Appl. Opt. 37, 3295 (1998).

[16] T. Pohl, T. Pattard, and J. M. Rost, Phys. Rev. Lett. 94, 205003 (2005).

[17] T. Pohl, T. Pattard, and J. M. Rost, Phys. Rev. A 70, 033416 (2004). 